\title{
Análise de diferentes sistemas adesivos em estudos in vitro: uma revisão
}

Análisis de diferentes sistemas adhesivos en estudios in vitro: una revision

Analysis of different adhesive systems in in vitro studies: a review

Kaique de Freitas MATOS ${ }^{1}$

Luciana Quesado de LAVOR $^{1}$

Natasha Muniz FONTES ${ }^{2}$

${ }^{1}$ Curso de graduação em Odontologia, UNILEÃO - Centro Universitário Dr. Leão Sampaio, 63040-405 Juazeiro do Norte - CE, Brasil

${ }^{2}$ Mestre e especialista em ortodontia pela universidade São Leopoldo Mandic Campinas-SP; Especialista em Dentística Restauradora

Universidade São Leopoldo Mandic, Professora do Centro Universitário Católica de Quixadá, UNICATÒLICA, 63900-257 Quixadá - CE, Brasil

\section{Resumo}

Introdução: Os adesivos dentários são materiais imprescindíveis as técnicas restauradoras adesivas atuais. Podem ser do tipo convencional, autocondicionante ou mais atualmente do tipo universal, ambos com diferentes passos para diferentes aplicabilidades clínicas. Sabe-se que dúvidas são existentes quanto a melhor indicação de materiais restauradores assim como sua efetividade clínica. Objetivo: O objetivo do presente trabalho foi analisar por meio de uma revisão de literatura estudos realizados com diferentes tipos de adesivos quanto a sua resistência a tração, pigmentação, aumento da longevidade da restauração e indicação. Materiais e Métodos: Foram selecionados 31 artigos por meio de uma busca nas bases de dados do PubMed, Scielo e Google acadêmico, entre os anos de 2008 e 2018. Pesquisas experimentais realizadas in vitro. Foram identificados 16 adesivos de diferentes marcas amplamente testados e listados na literatura. As metodologias utilizadas nos diferentes estudos incluíram corpos de prova submetidos a diferentes testes de efetividade adesiva, tais como: resistência à tração, adesão, cisalhamento, infiltração e longevidade. Resultados e Conclusão: Percebe-se que a escolha do adesivo dentário parte do caso clínico proposto, no qual fatores como o substrato dentário, localização, presença de tecido cariado, umidade e protocolo clínico são fundamentais para seleção, partindo de tal pressuposto a efetividade pode ser aumentada pelo uso de substâncias na lavagem, como também a utilização de materiais resinosos, agentes desproteinizantes e antioxidantes.

Descritores: Cimentos Dentários; Protocolos Clínicos; Esmalte Dentário; Dentina.

\section{Abstract}

Introduction: Dental adhesives are essential materials for current adhesive restorative techniques. They can be conventional, self-conditioning or more currently universal, both with different steps for different clinical applications. It is known that doubts exist as to the best indication of restorative materials as well as their clinical effectiveness. Objective: The objective of this study was to analyze, by means of a literature review, studies performed with different types of adhesives regarding their tensile strength, pigmentation, increased longevity of the restoration and indication. Materials and Methods: 31 articles were selected through a search in the PubMed, Scielo and Google academic databases, between the years 2008 and 2018 . Experimental research conducted in vitro. Sixteen adhesives from different brands widely tested and listed in the literature were identified. The methodologies used in the different studies included specimens submitted to different adhesive effectiveness tests, such as: tensile strength, adhesion, shear, infiltration and longevity. Results and Conclusion: It is noticeable that the choice of dental adhesive starts from the clinical case proposed, in which factors such as dental substrate, location, presence of carious tissue, moisture and clinical protocol are fundamental for selection, based on this assumption the effectiveness can be increased by the use of substances in washing, as well as the use of resin materials, deproteinizing agents and antioxidants.

Descriptors: Dental Cements; Clinical Protocols; Dental Enamel; Dentin.

\section{Resumen}

Introducción: Los adhesivos dentales son materiales esenciales para las actuales técnicas de restauración con adhesivos. Pueden ser convencionales, de autocondicionamiento o más actualmente universales, ambos con diferentes pasos para diferentes aplicaciones clínicas. Se sabe que existen dudas sobre la mejor indicación de los materiales de restauración, así como sobre su eficacia clínica. Objetivo: El objetivo de este estudio fue analizar, mediante una revisión de la literatura, los estudios realizados con diferentes tipos de adhesivos en cuanto a su resistencia a la tracción, pigmentación, aumento de la longevidad de la restauración e indicación. Materiales y métodos: Se seleccionaron 31 artículos mediante una búsqueda en las bases de datos académicas PubMed, Scielo y Google, entre los años 2008 y 2018 . Investigación experimental realizada in vitro. Se identificaron dieciséis adhesivos de diferentes marcas ampliamente probados y listados en la literatura. Las metodologías utilizadas en los diferentes estudios incluyeron muestras sometidas a diferentes pruebas de eficacia adhesiva, como: resistencia a la tracción, adhesión, cizallamiento, infiltración y longevidad. Resultados y Conclusión: Es notable que la elección del adhesivo dental parte del caso clínico propuesto, en el que factores como el sustrato dental, la ubicación, la presencia de tejido cariado, la humedad y el protocolo clínico son fundamentales para la selección, partiendo de este supuesto la eficacia puede incrementarse mediante el uso de sustancias en el lavado, así como el uso de materiales de resina, agentes desproteinizantes y antioxidantes.

Descriptores: Cementos Dentales; Protocolos Clínicos; Esmalte Dental; Dentina.

INTRODUÇÃO

A evolução significativa que ocorreu na área dos sistemas adesivos, em especial nos últimos anos, proporcionou uma completa modificação na prática da Odontologia restauradora. Os preparos cavitários tradicionais, delineados com base na anatomia dental e realizados de maneira padronizada com dimensões desnecessariamente grandes, estão sendo cada vez mais substituídos por procedimentos restauradores menos invasivos, graças ao desenvolvimento dos materiais adesivos $^{1,2}$.
Nos últimos anos os materiais restauradores passaram por uma série de

desenvolvimentos, e com o avanço da tecnologia é notório a diversidade de materiais disponíveis no mercado odontológico, produtos esses com maior capacidade estética e funcional. A durabilidade de procedimentos restauradores depende da união do material restaurador e estrutura dentária, visto que a falta de adesão é um dos maiores problemas durante esse tipo de procedimento ${ }^{3}$. 
Sendo a união dos materiais restauradores a estrutura dentária de grande importância. Diante disso, estudos frequentes são realizados com intuito de melhorar as propriedades dos adesivos dentinários, dado que a falta de adesão pode contribuir na infiltração marginal e sensibilidade pósoperatória. No momento atual, encontram-se dois tipos de sistemas adesivos, os adesivos autocondicionantes que não necessitam de um condicionamento ácido prévio e os adesivos convencionais que precisam de um condicionamento ácido prévio ${ }^{4,5}$.

Os adesivos autocondicionantes podem ser encontrados do tipo, um passo onde encontra-se em um único frasco e em dois passos em que o primer é separado do adesivo, ao serem utilizados não existe a fase de condicionamento, com isso não tem aplicação do ácido e nem a necessidade de lavagem e secagem, assim sendo a smear layer não é removida, apenas modificada ${ }^{6}$.

Os sistemas adesivos convencionais, também chamados de condicionamento total ou total etch, removem a smear layer, apresentam boa união ao esmalte e a dentina, uma vez que na estrutura de esmalte o mesmo aumenta a área e energia de superfície, enquanto que na dentina expõe fibras colágenas, porém deve haver o cuidado na sua aplicação, uma vez que o condicionamento excessivo pode gerar falhas como a nanoinfiltração, assim como a falha no controle de umidade pode colapsar as fibras colágenas prejudicando a adesão $0^{3,7}$.

Para o sucesso e longevidade das restaurações diretas as estruturas de reforço, o tecido remanescente, quantidade e qualidade desse tecido, assim como a composição do adesivo, evitam condições como a microinfiltração, danos a polpa, deficiência na adesividade da restauração, sensibilidade pósoperatória. Por tais justificativas é de suma importância que o cirurgião dentista tenha conhecimentos sobre a composição dos adesivos, forma de uso, assim como sua interação com diferentes tipos de substratos dentais em diferentes condições clinicas ${ }^{8}$.

O objetivo do presente trabalho foi analisar por meio de uma revisão de literatura estudos realizados com diferentes tipos de adesivos quanto a sua resistência a tração, pigmentação, aumento da longevidade da restauração e indicação.

MATERIAL E MÉTODO

Foram selecionados 34 artigos por meio de uma busca nas bases de dados do PubMed, Scielo e Google acadêmico, entre os anos de
2008 e 2018. Pesquisas experimentais realizadas in vitro. Foram identificados 16 adesivos de diferentes marcas amplamente testados e listados na literatura. As metodologias utilizadas nos diferentes estudos incluíram corpos de prova submetidos a diferentes testes de efetividade adesiva, tais como: resistência à tração, adesão, cisalhamento, infiltração e longevidade.

RESULTADOS

- Revisão da Literatura

Para que os procedimentos restauradores possuam uma maior durabilidade, é indispensável uma união eficaz entre o material restaurador e a estrutura dentária, com isso, observou-se a necessidade da utilização de materiais que proporcionassem uma boa adesão, e então em 1955 com Buonocore deu início a utilização do sistema adesivo, no qual o mesmo usou ácido fosfórico $85 \%$ durante um tempo de 30 segundos, e através desse condicionamento ácido obteve boa interação entre o esmalte dentário e a resina acrílica ${ }^{3,9}$.

O princípio fundamental de adesão à estrutura dentária está baseado em um processo de troca, no qual minerais são removidos dos tecidos dentários e então substituídos por monômeros resinosos. Este processo envolve duas fases. Enquanto a primeira fase consiste na remoção do cálcio e criação de porosidades tanto em esmalte quanto em dentina; a segunda, denominada hibridização, envolve a penetração e polimerização dos monômeros no interior das porosidades criadas $^{2,10}$. Enquanto este embricamento mecânico representa um prérequisito para a obtenção de uma adesão adequada, o potencial benefício de uma adesão química adicional entre os monômeros resinosos funcionais presentes nos sistemas adesivos e os componentes da estrutura dental têm merecido atenção da literatura. Uma interação química com as estruturas dentárias seria particularmente importante para aumentar a durabilidade adesiva ${ }^{2,10,11}$.

O sistema adesivo é utilizado tanto em dentina como em esmalte. Sendo a dentina um tecido composto de água, substância orgânica e em sua maioria substância inorgânica, há a presença dos túbulos dentinários, e quando se aplica o sistema adesivo sobre esse tecido, esses túbulos dentinários aumentam de diâmetro e alteram a permeabilidade, facilitando assim a adesão, porém por ser um tecido com diferentes características morfológicas, a adesão é considerada menos provável quando comparada ao esmalte. Já o esmalte é um 
tecido altamente mineralizado, composto por hidroxiapatita em sua grande maioria, e por material orgânico e água em proporções menores, com isso a adesão em esmalte é tido como mais efetiva e duradoura quando contraposto a dentina ${ }^{9}$.

No mercado odontológico existem variados tipos de sistemas adesivos de diferentes marcas. Hoje basicamente os adesivos estão divididos em convencionais e autocondicionantes, sabido a presença do tipo universal, adesivo esse pouco abordado na literatura $^{6,12}$

\section{- Adesivos de condicionamento total ou convencionais}

Os sistemas adesivos de condicionamento total também conhecidos como sistema adesivo convencional ou total etch, atuam removendo a smear layer através do uso do ácido fosfórico de 30 a $40 \%$ usado na dentina e no esmalte, no esmalte o ácido irá desmineralizar resultando em um aumento da energia de superfície e no aumento da área de superfície, enquanto que na dentina irá expor fibras colágenas e remover resquícios de hidroxiapatita ${ }^{7}$.

Existem sistemas total etch de 3 ou 2 passos. Os de 3 passos possuem ácido, primer e bond separadamente, no qual é utilizado o ácido fosfórico por 15 a 30 segundos em esmalte e no máximo 15 segundos em dentina, seguido da lavagem para remoção de possíveis resquícios do ácido e smear layer, secagem, aplicação do primer e finalizando com a aplicação do bond. O adesivo de 2 passos segue a mesma sequência sendo a sua diferenciação no primer e bond que são juntos, para melhor controle de umidade das fibras colágenas expostas ${ }^{8}$.

Esse tipo de sistema adesivo requer precauções como o controle do tempo de exposição, uma vez que esse tempo ultrapassado gera nanoinfiltração e consequentemente aumenta 0 risco de sensibilidade dentaria do paciente, como também o controle de umidade deve ser satisfatório uma vez que as fibras colágenas são expostas para a penetração do adesivo e da resina composta, fazendo sua microretenção, sendo que a secagem excessiva gera 0 colapso das fibras colágenas e consequentemente falha adesiva ${ }^{3}$.

Estudos evidenciaram que os adesivos convencionais apresentaram menor resistência da interface adesiva se comparado aos autocondicionantes, isso é explicado pela sua degradação que são distintas. Porém evidenciaram que a adesão dos sistemas adesivos convencionais é maior, e tanto os autocondiconantes quanto os de condicionamento total formam uma camada híbrida uniforme e espessa ${ }^{13}$.

- Adesivos autocondicionantes.

Esse tipo de sistema tem sua camada híbrida por meio de um primer acidulado, manobra que dispensa os cuidados com o isolamento da umidade requerente aos adesivos convencionais, pois à medida que a camada é desmineralizada o adesivo é então penetrado evitando que as fibras colágenas colabem, sendo assim uma técnica mais sensível se comparada ao de condicionamento total, como uma técnica com menor número de passos uma vez que a etapa de lavagem e secagem está ausente ${ }^{14}$.

Os adesivos autocondicionantes apresentam menor risco à nanoinfiltração uma vez que, à medida que desmineralizam se impregna o adesivo. São compostos pelo primer, monômeros dissolvidos em água, acetona ou álcool de características hidrofílicas que permitem a difusão pela dentina umedecida, enquanto que o adesivo é composto por monômeros resinosos hidrofóbicos como o Bis-GMA ${ }^{15}$.

Também conhecidos por self-etch podem se apresentar em 2 passos, primer acidulado e adesivo, ou 1 passo quando todos os componentes estão presentes em um único frasco ${ }^{4}$. Nesse sistema a smear layer não é removida e sim incorporada a interface adesiva, fator que previne a reidratação imediata que desencadeia movimentação de fluidos dentinários resultando na sensibilidade pós-operatória ${ }^{8}$.

A adesão à estrutura dentinária é superior à estrutura de esmalte, tal característica é dada pela estrutura e tamanho dos cristais de hidroxiapatita que o tornam menos sensíveis a interação com o primer acidulado. Tal aspecto pode ser sanado na criação de retenções na estrutura de esmalte realizadas com o condicionamento prévio com ácido fosfórico ${ }^{16}$.

DISCUSSÃO

Os adesivos dentários consistem em um artifício de suma importância para técnica restauradora adesiva, técnica essa preferível para os pacientes quanto aos profissionais por suas características estéticas e controle de presa, nesse âmbito se desenvolveram diferentes técnicas e adesivos com o objetivo de suprir a demanda clínica ${ }^{17}$. Sabe-se que há recorrentes dúvidas por parte da comunidade científica a respeito da efetividade dos sistemas 
adesivos autocondicionantes, em detrimentos os de condicionamento total, no que se refere a sua ação em esmalte, dentina, assim como a incorporação de substâncias, como a clorexidina no seu aumento de efetividade. Portanto, foram comparados na literatura, 16 adesivos distintos de diferentes passos e ações, assim como aplicados em diferentes substratos.

O Adper Scotchbond Multi-Purpose ${ }^{\circledR}$, é um adesivo de condicionamento total de 3 passos. Para Zappelini ${ }^{18}$ esse tipo de sistema é mais efetivo na resistência de união ao substrato e que apresenta um melhor selamento marginal da dentina, quando comparado a sistemas adesivos convencionais de 2 passos (Adper Single Bond $2 \AA$ ) e autocondicionantes ${ }^{19}$. Segundo Isolan et al. ${ }^{19}$ o adesivo apresenta menor microtensão e força de adesão, quando comparado ao Adper Single Bond $2 \AA$, porém a resistência de união se mostra semelhante, contrariando os estudos de Zappelini ${ }^{18}$. A aplicação da resina flow melhora a resistência de união, porém de forma não significativa, pois seu uso como camada adicional aumenta as chances de fraturas adesivas, como também não melhora o desempenho adesivo ${ }^{7,20}$.

O Prime \& Bond NT®, é um adesivo de condicionamento total de 2 passos, Muller et al. $^{21}$ observaram que a força de adesão deste é menor quando comparada com o Single Bond Universal $\AA^{\circledR}$, porém não é alterada pelo substrato diferentemente do segundo. Quanto a utilização da clorexidina $2 \%$ após a etapa de ataque ácido, Grande ${ }^{15}$ observou interferência na resistência de união e não na degradação ao longo do tempo. Em contrapartida, Ricci ${ }^{22}$ demonstrou que a clorexidina não interferiu no desempenho adesivo, e que após 18 meses há uma redução desse desempenho adesivo, na qual a clorexidina desacelera esse processo.

O XP Bond® $\mathrm{B}$, um adesivo convencional de 2 passos, foi estudado por Kimmes et al. ${ }^{23}$ que demonstraram que 0 uso por tempo prolongado além do recomendado pelo fabricante, em esmalte, não interfere na força de adesão, enquanto que em dentina há o aumento dessa força. Machado ${ }^{24}$ ao testar diferentes temperaturas, observou que o aquecimento a temperatura corporal $\left(37^{\circ}\right)$ aumenta a resistência adesiva em dentina, 24 horas após o uso, assim como após 180 dias. Já Castillo et $\mathrm{al}^{4}{ }^{4}$ observaram que a dentina úmida tem melhor desempenho quanto a resistência a tração, quando comparado a dentina seca.

Estudos de Ricci ${ }^{22}$ sobre Excite DSC $\AA$, um adesivo do tipo convencional de 2 passos, mostrou que o material possui o mesmo desempenho em dentes permanentes e decíduos quanto a resistência de união, pois o uso da clorexidina $2 \%$ na etapa de lavagem eleva essa resistência, assim como desacelera a degradação da interface adesiva. Neves et al. ${ }^{5}$ sobre o tratamento da dentina com diferentes substâncias, observou que o etanol associado ou não com a clorexidina, aumenta a resistência de união quando comparado a lavagem com água, com ou sem associação a clorexidina.

Bravo et al. ${ }^{25}$ observaram que 0 adesivo convencional de 2 passos Scothbond $1 \mathrm{XT} \AA$, apresentou elevada resistência adesiva no período de 3 e 6 meses frente ao uso da clorexidina na dentina. Já quanto a permeabilidade dentinária, Rodrigues et al. ${ }^{26}$ demonstraram que 0 uso de uma camada hidrófoba de resina reduz seus valores, assim como o aumento da pressão pulpar origina defeitos estruturais.

Spazzin et al. $^{27}$ ao estudar Prime \& Bond $\AA$, um total-etch de 2 passos, encontraram resistência de união maior quando em dentina umedecida, porém com falha predominante do tipo adesiva, seja em dentina seca ou úmida. Neves et al. ${ }^{5}$ relatam que a utilização de etanol ou a sua associação com clorexidina, aumenta a resistência de união quando comparado a lavagem com água.

Dias $^{8}$ em estudo comparativo entre o Clearfil S3 Bond $\AA$, Futurabond $M \AA$ e Optibond FL® observou que o Clearfil Se Protect ${ }^{\circledR}$ (SEP), adesivo autocondicionante de 2 passos, apresentou maior resistência adesiva à dentina ao teste de microtração, maior força de adesão nos períodos de 24 horas a 3 meses, mas a força manteve acentuada diferença aos demais com 3 meses, além de ser o único que não foi sensível a degradação hidrolítica. Já Krifka et al. $^{28}$ mostraram que SEP apresenta maior resistência ao cisalhamento em esmalte quando comparado a dentina, como também que a resistência em esmalte só é inferior a do Syntac Assortment $\AA$ entre os 4 adesivos testados, à medida que a dentina é inferior somente ao Ibond Gluma Inside ${ }^{\circledR}$ e igual ao Adaper Prompt L-Pop®.

Garcia et al. ${ }^{29}$, em seu estudo comparativo entre sistemas adesivos quanto à ação em esmalte e dentina, verificou que o Adper SE Plus ${ }^{\circledR}$, adesivo autocondicionante de 2 passos, apresenta ação similar aos demais em esmalte e ação superior em dentina. Já Sampaio ${ }^{13}$ observou que a resistência adesiva à dentina, se apresentou como 0 menos resistente após um período de 24 horas, ao passo que na comparação após 6 meses foi o mais resistente, porém a diferença não é significativa. Em comparação com o Adper 
Single Bond $2 \AA$, em relação ao selamento das interfaces, realizado por Aguiar et al. $^{30} \mathrm{o}$ resultado foi semelhante.

System Adhesive $\mathrm{P} 90 \AA$, também consiste em um sistema adesivo autocondicionante de 2 passos, utilizado nos estudos de Garcia et al. ${ }^{29}$ e Sampaio ${ }^{13}$. Esse adesivo em específico, apresentou adesividade em esmalte semelhante aos demais, e em dentina inferior apenas ao Adper SE Plus ${ }^{\circledR}$, ao passo que à resistência a adesão após 24 horas, só foi superior ao Adper SE Plus $\AA$, já que após 6 meses dos 4 testados, ele consistiu no terceiro com menor resistência.

O Clearfil SE Bond $\AA$, também consiste em um adesivo autocondicionante de 2 passos, estudado por Goes et al. ${ }^{20}$ que apontaram maior resistência de união entre todos os adesivos testados, porém quando utilizado com a resina flow essa resistência é aumentada. Mine et al. ${ }^{31}$ corroboram tal experimento e acrescentam que as fraturas mais recorrentes desse tipo de adesivo, são falhas mistas que ocorrem na interface da restauração e parcialmente na resina. Já Sampaio ${ }^{13}$ analisou essa resistência em diferentes períodos, entretanto no período de 24 horas foi 0 que apresentou melhor resistência de união, porém após 6 meses o Adper SE Plus $₫$ obteve valores superiores, mas com semelhanças estatísticas. Macedo et al. ${ }^{32}$ apontam que ambos tem ações semelhantes, enquanto que Andrade et al. ${ }^{17}$ verificaram que a resistência de união em esmalte é próxima ao de um adesivo de condicionamento total e superior em dentina. Porém, quando comparado a outros adesivos autocondicionantes, seja de 1 ou 2 passos, apresenta melhores valores em ambos os substratos. Essa mesma conclusão também foi obtida por Isolan et al. ${ }^{19}$ e Kimmes et al. ${ }^{23}$. Contudo, para Kimmes et al. ${ }^{23}$, Xeno V® gerou a mesma resistência que o Clearfil SE Bond®. A utilização do digluconato de clorexidina a $2 \%$, assim como a utilização em dentes afetados por cárie não exerceu interferência na sua ação $0^{6,14}$.

Adper Single Bond $2 \AA$ é um self-etch de 2 passos, amplamente estudado, quanto a força de adesão. No estudo de Isolan et al. ${ }^{19}$ apresentou os melhores resultados em esmalte e dentina. Frente a resistência de união, Andrade et al. ${ }^{17}$ observaram ser a maior quando em esmalte, e inferior apenas ao Clearfil SE® em dentina, dos 4 adesivos testados, em contrapartida Sampaio ${ }^{13}$ verificou que não há diferença estatística quando em dentina em relação a outros materiais. Macedo et al. ${ }^{32}$ corroboram tal afirmativa e acrescenta que as principais falhas nesse tipo de adesivo são do tipo adesiva. Em relação ao selamento marginal, Zappelini ${ }^{18}$ relata que é efetivo em dentina, enquanto Aguiar et al. $^{30}$ apresentam como semelhante ao Adper SE Plus ${ }^{\circledR}$ e que ambos apresentam infiltração nas paredes axiais e gengivais. $\mathrm{O}$ aquecimento do adesivo a temperatura corporal, mostrou aumentar a resistência de união quando comparado ao mesmo em temperatura ambiente, assim como o uso da resina flow também o faz ${ }^{20,24}$. A clorexidina $2 \%$ utilizada previamente ao adesivo, não interfere negativamente na resistência de união, desacelerando a degradação da interface adesiva ${ }^{5,14,15,22,}$. Segundo Ribeiro et al. $^{33}$, quanto ao teste da associação de agente desproteinizante e agente antioxidante, mostrou que o hipoclorito de sódio $10 \%$ associado ao ácido ascórbico aumentaram a resistência de união do Adper Single Bond $2 \circledast$.

Clearfil S3 Bond Plus, consiste em um adesivo autocondicionante de 1 passo. Segundo Dias $^{8}$ sua resistência adesiva diminui no período de 24 a 3 meses, o que diverge do SEP. Kimmes et al. ${ }^{23}$ apontam que todos os adesivos autocondicionantes, apresentaram menor força de adesão ao esmalte quando comparado aos de condicionamento total.

O Adper Prompt LPOP®, também consiste em um adesivo autocondicionante de 1 passo, estudado por Andrade et al. ${ }^{17}$ que observaram que a sua resistência adesiva ao esmalte e a dentina são menores quando comparado a um adesivo de condicionamento total. Krifka et al. ${ }^{28}$ ao avaliar a resistência de união ao cisalhamento em esmalte e dentina de dentes decíduos, verificou que 0 LPOP apresentou o $2^{\circ}$ menor resultado, sendo que a aplicação da resina flow aumenta a resistência de união. Quando estendido o tempo de uso além do recomendado pelo fabricante, a força de ligação ao esmalte aumenta quando comparado ao uso no tempo recomendado, assim como o uso da clorexidina tende a melhorar a adesão a dentina nos períodos de 72 horas, 3 meses e 6 meses $^{20,23,25}$.

Outro adesivo autocondicionante de 1 passo é o Easybond®. Para Mine et al. $^{31}$ a principal falha em esmalte é do tipo adesiva, enquanto que em dentina as falhas do tipo mista possuem maior prevalência. Já sua adesão a dentina é inferior aos dois adesivos testados, Adper Scotchbond SE $\AA$ e Clearfil SE Bond $\AA$, com valores reduzidos pelo uso de uma camada de resina hidrófoba ${ }^{26}$.

O sistema adesivo Optibond Solo Plus ${ }^{\circledR}$ não apresentou diferença estatística na resistência de união à dentina em comparação a 
outros adesivos autocondicionantes. No estudo de Macedo et al. ${ }^{32}$ apresentou falhas na sua maioria do tipo mista. Já Kimmes et $a{ }^{23}$, o classifica como adesivo com etapa de lavagem, no qual ao teste do tempo de ação prolongado demonstrou que em esmalte não há alteração na força de união, enquanto que na dentina há redução.

Já o Single Bond Universal®, é um adesivo do tipo universal que pode ser usado com condicionamento ácido prévio ou não. Zappelini $^{18}$ demonstrou que 0 ataque ácido prévio aumenta a resistência de união, como também causa menor infiltração em esmalte; já em dentina não apresenta diferença significante na ausência do condicionamento ácido. Em contrapartida, Fernandes $^{12}$ relata que 0 condicionamento total, assim como a utilização da clorexidina não altera a resistência de união, porém a mesma resultou em um número maior de falhas. Para Bravo et al. ${ }^{25}$, a utilização da clorexidina promove o aumento da força de união no período de 3 e 6 meses após a aplicação. A fricção do adesivo quando utilizado como autocondicionante não melhora a sua efetividade, ao passo que na ausência de tecido cariado a resistência de união é superior a presença de dentina cariada ${ }^{21,34}$.

CONCLUSÃO

Percebe-se que a escolha do adesivo dentário parte do caso clínico proposto, nos fatores como o substrato dentário, localização, presença de tecido cariado, umidade e protocolo clínico são fundamentais para seleção, partindo de tal pressuposto a efetividade pode ser aumentada pelo uso de substâncias na lavagem, como também a utilização de materias resinosos, agentes desproteinizantes e antioxidantes.

\section{REFERÊNCIAS}

1. Alex G. Universal adhesives: the next evolution in adhesive dentistry? Compend Contin Educ Dent. 2015;36(1):15-26.

2. Arinelli AMD, Pereira KF, Prado NAS, Rabello TB. Sistemas adesivos atuais. Rev. bras. odontol. 2016;73(3):242-46.

3. Martins GC, Franco AP, Godoy ED, Maluf DR, Gomes JC, Gomes OM. Adesivos dentinários. RGO Rev Gauch Odontol. 2008;56(4): 429-36.

4. Castillo KA, Costa SR, Barros RM, Guerisoli DM, Figueiredo JL. Resistência a tração de um sistema adesivo em dentina seca e em dentina. Arch Health Invest. 2013;2(4):11-7.

5. Neves TP, Leandrin TP, Tonetto MR, Andrade MF, Campos EA. Resistência de união à microtração de sistemas adesivos "condicionae-lava" de dois passos: efeito de diferentes tratamentos da superfície dentinária condicionada. Rev Odontol UNESP. 2017; 46(3):131-37.

6. Silva CM, Lima AR, Azavedo CS, Andrade AP, Matos AB. Estudo da adesão de sistema autocondicionante utilizado em dentina hígida e afetada por cárie. Rev Assoc Paul Cir Dent. 2013;67(3):194-97.

7. Carvalho CL. Estudo in vitro das forças de adesão à dentina de um Sistema Adesivo Universal pela técnica Etch-and-rinse quando aplicada uma camada adicional de resina hidrofóbica. Lisboa: Universidade de Lisboa; 2016.

8. Dias CBGSR. Estudo in vitro da resistência adesiva à dentina, por microtração, de sistemas adesivos self-etch e universais [dissertação]. Almada, Portugal: Egas Moniz - Cooperativa de Ensino Superior, CRL; 2016.

9. Sousa JHP, Moro AFV. Solventes do Primer: revisão de literatura. Rev Bras Odontol. 2014; 71(1):80-4.

10. Van Meerbeek B, De Munck J, Yoshida Y, Inoue $S$, Vargas $M$, Vijay $\mathrm{P}$, Van Landuyt $\mathrm{K}$, Lambrechts P, Vanherle G. Buonocore memorial lecture. Adhesion to enamel and dentin: current status and future challenges. Oper Dent. 2003; 28(3):215-35.

11. Van Meerbeek B, Yoshihara K, Yoshida $Y$, Mine A, De Munck J, Van Landuyt KL. State of the art of self-etch adhesives. Dent Mater. $2011 ; 27(1): 17-28$.

12. Fernandes GL. Resistência de união de sistema adesivo universal à dentina com diferentes protocolos de união [monografia]. Araçatuba: Faculdade de Odontologia de Araçatuba, UNESP; 2013.

13. Sampaio RKD. Adesão de materiais resinosos com e sem silorano à dentina [dissertação]. Londrina: Universidade do Norte do Paraná, UNOPAR; 2011.

14. Bengtson $C R$, Bengtson $A L$, Bengtson NG, Turbino ML. Efeito da Clorexidina 2\% na Resistência de União de Dois Sistemas Adesivos à Dentina Humana. Pesqui bras odontopediatria clín integr. 2008;8(1):51-6.

15. Grande RS. Avaliação da aplicação da clorexidina na resistência de união de sistemas adesivos convencionais [dissertação] Ponta Grossa: Universidade estadual de Ponta Grossa (UEPG); 2008.

16. Sousa MNG. Estudo in vitro: adesão ao esmalte de dois sistemas adesivos self-etch [dissertação]. Lisboa: Universidade de Lisboa; 2014.

17. Andrade AP, Shimaoka AM, Russo EM, Carvalho RC. Estudo comparativo da resistência de união de sistemas adesivos autocondicionantes com diferentes $\mathrm{pHs}$ aplicados ao esmalte e à dentina. RGO (Porto 
Alegre). 2008;56(2):115-19.

18.Zappelini HV. Influência do sistema adesivo na microinfiltração e resistência de união adesiva [monografia]. Florianópolis: Universidade Federal de Santa Catariina (UFSC); 2014.

19. Isolan CP, Valente LL, Münchow EA, Basso GR, Pimentel AH, Schwantz JK, et al. Bond strength of a universal bonding agent and other contemporary dental adhesives applied on enamel, dentin, composite, and porcelain. Appl Adhes Sci. 2014;2(25):1-10.

20. Goes MF, Giannini M, Hipólito VD, Carrilho MR, Daronch M, Rueggeberg FA. Microtensile Bond Strength of Adhesive Systems to Dentin with or without Application of an Intermediate Flowable Resin Layer. Braz Dent J. 2008;19(1):51-6.

21. Müller C, Rosa GC, Teixeira GS, Krejci I, Bortolotto T, Susin AH. Effect of caries-affected dentin on one-step universal and multi-step etch-and-rinse adhesives' bond strength. Rev Odontol UNESP. 2017;46(5):273-77.

22. Ricci HA. Influência do substrato, dentes decíduos e permanentes, e da aplicação de clorexidina na resistência de união de sistemas adesivos à dentina [dissertação]. Araraquara: Faculdade de Odontologia de Araraquara, UNESP; 2008.

23. Kimmes NS, Barkmeier WW, Erickson RL, Latta MA. Adhesive bond strengths to enamel and dentin using recommended and extended treatment times. Oper Dent. 2010;35(1):112-19.

24. Machado P. Avaliação do efeito da temperatura dos sistemas adesivos na resistência de união à dentina. Santa Maria: Universidade Federal de Santa Maria, UFSM; 2012.

25. Bravo C, Sampaio CS, Hirata R, PuppinRontani RM, Mayoral JR, Giner L. In-vitro comparative study of the use of $2 \%$ chlorhexidine on microtensile bond strength of different dentin adhesives: a 6 months evaluation. Int J Morphol. 2017;35(3):893-900.

26. Rodrigues S, Oliveira N, Chasqueira F, Portugal J, Arantes-Oliveira S. Permeabilidade dentinária e morfologia da interface adesiva de diferentes sistemas adesivos. Rev Port Estomatol Med Dent Cir Maxilofac. 2015;56(1):42-50.

27. Spazzin AO, Júnior BC, Moraes RR, Mesquita MF. Adesão à dentina úmida e seca: resistência de união à microtração e infiltração marginal. Rev Odontol UNESP. 2008;37(1): 91-6.

28. Krifka $S$, Börzsönyi $A$, Koch $A$, Hiller KA, Schmalz G, Friedl $\mathrm{KH}$. Bond strength of adhesive systems to dentin and enamel-human vs. bovine primary teeth in vitro. Dent Mater. 2008;24(7):888-94.

29. Garcia RN, Alvarez AE, Dias CE, Mazaro MA, Firmo $\mathrm{T}$, Stuker $\mathrm{H}$, et al. Avaliação da resistência de união de sistemas restauradores contemporâneos em esmalte e dentina. RSBO. $2011 ; 8(1): 60-7$.

30. Aguiar A, Seixas GF, Simões TC, Fernandes ABF, Busato PMR, Moura SK. Avaliação do selamento marginal promovido por sistemas adesivos aplicados em esmalte e dentina. J Oral Invest. 2014;3(1):4-9.

31. Mine A, De Munck J, Cardoso MV, Van Landuyt $\mathrm{KL}$, Poitevin A, Kuboki $\mathrm{T}$ et al. Bonding effectiveness of two contemporary self-etch adhesives to enamel and dentin. J Dent. 2009;37(11):872-83.

32. Macedo DR, Dorini AL, Mendonça JS. Influência de sistemas adesivos autocondicionantes na resistência de união da resina composta à dentina. Rev Bras Pesq Saúde. 2010;12(1):47-51.

33. Ribeiro Al, Dantas DC, Guênes GM, Araújo RK, Cyrillo CC, Braz R. Ação dos agentes desproteinizantes e antioxidantes sobre a resistência de união à microtração de sistemas adesivos convencionais. RGO, Rev gaúch odontol. 2011;59(2): 221-27.

34. Irmak Ö, Yaman BC, Orhan EO, Ozer F, Blatz MB. Effect of rubbing force magnitude on bond strength of universal adhesives applied in selfetch mode. Dent Mater J. 2018;37(1):139-45.

CONFLITO DE INTERESSES

Os autores declaram não haver conflitos de interesse

AUTOR PARA CORRESPONDÊNCIA

\section{Natasha Muniz Fontes}

Centro Universitário Católica de Quixadá UNICATÓLICA

Avenida Desembargador Moreira, 760 sala 110960170 000 Aldeota, Fortaleza - CE, Brasil

Telefone: +55 (85) 996319546

E-mail: natashafontes@hotmail.com 